ESAIM: PROCEEDINGS, October 2007, Vol. 22, 175-180

Gabriel Caloz \& Monique Dauge, Editors

\title{
MULTILEVEL GRADIENT-BASED METHODS IN AERODYNAMIC SHAPE DESIGN
}

\author{
Massimiliano Martinelli ${ }^{1}$ And Francois BeuX ${ }^{1}$
}

\begin{abstract}
A gradient-based method coupled with a multilevel approach is proposed for shape design in aerodynamics. This method extends an existing multilevel gradient-based formulation to another type of control subspaces, i.e. considering another set of subparametrisations and prolongation operators. More precisely, Bézier control points associated with the property of degree elevation are involved instead of shape grid-points coordinates and polynomial interpolation. The good behaviour of the new formulation is demonstrated on a classical 2D nozzle inverse problem considering an adjoint formulation as well as an approximate gradient associated to a one-shot method.
\end{abstract}

Résumé. Une méthode de type gradient associée à une approche multiniveau est proposée dans le cadre de problèmes d'optimisation de forme en aérodynamique. Cette méthode généralise une formulation déjà existante de méthode de gradient multiniveau considérant un autre type de sousespace de contrôle, c'est à dire considérant un autre ensemble de sous-paramétrisation et d'opérateur de prolongement. Plus précisement, des points de contrôle de Bézier associés à la propriété d'élévation de degré sont utilisés au lieu des coordonnées des points du maillage sur la frontière et une interpolation polynomiale. Le bon comportement de la nouvelle formulation est illustré pour le cas d'un problème inverse classique d'une tuyère bidimensionelle considérant aussi bien une méthode de l'adjoint que le calcul d'un gradient approché associé à une méthode de type "one-shot".

\section{INTRODUCTION}

In this study, we are interested in gradient-like methods for shape optimisation problems in aerodynamics. More particularly, we consider the computation of a discrete gradient, i.e. the differentiation is performed after a complete discretisation of the governing equations. In this context, a natural choice of parametrisation is to consider the coordinates of the grid-points localised on the shape which should be optimised. Indeed, this kind of parametrisation allows a direct correlation with the explicit representation of the shape in the discrete cost functional. Nevertheless, in this case, non-smooth profiles can appear during the shape optimisation process. This phenomenon, particularly critical for shape grid-points parametrisation, can be also linked with a regularity loss of the gradient with respect to the control variables, already verified in the continuous case (see, e.g. [3] or [6]). Thus, to avoid high frequencies in the discrete gradient involved in the shape updating, a preconditioning procedure should be applied. The necessity to define a smoothing operator can be also understood through the consistent approximation theory proposed by Polak (see [7]). A possible way to precondition is based on the inversion of a Laplace-Beltrami operator, which corresponds to a change of metric on which the minimisation is done [6,8]. Alternatively, in [3], an adequate gain of regularity for the gradient is obtained through the use of an additive multilevel approach. Note that multilevel concepts, in the context of gradient-like methods

${ }^{1}$ Scuola Normale Superiore di Pisa, Piazza dei Cavalieri, 7 - 56126 Pisa (Italy); e-mail: max.martinelli@sns.it \& fbeux@sns.it

(c) EDP Sciences, SMAI 2007 
for optimum shape design, have been initially ideated in [2]. In this approach, the minimisation is done alternatively on different subsets of control parameters according to multigrid-like cycles of multiplicative type. More particularly, using shape grid-point coordinates as design variables, a hierarchical parametrisation was defined considering different subsets of parameters extracted from the complete parameterisation, which can be prolongated to the higher level by linear mapping. This approach has been also defined to make the convergence rate of a gradient-based method almost independent of the number of control parameters.

Alternatively to shape grid-points, a polynomial representation of the shape is often used allowing a more compact description with only few control parameters. In particular, in [4], a multilevel approach is proposed considering as control parameters a set of Bézier control points. Nevertheless, this approach sensibly differs from the method defined in [2] since, in particular, the study was not focused on gradient-based methods.

In the present study, a new multilevel strategy based on the use of Bézier control points, but in the context of a gradient-based method, is proposed. Indeed, the present algorithm is grounded on some basic concepts yet proposed in [4], as, for instance, the degree elevation property of the Bézier curves, but on another hand, can be interpreted as a multilevel strategy as defined in [2], in which a particular prolongation operator is applied.

A first validation is proposed on a classical 2D nozzle inverse problem for inviscid flows.

\section{Gradient-Based method and multilevel approach}

The multilevel method, proposed in [2], is based on a change of control space. More precisely, let $E$ and $F$ be two Hilbert spaces and $P: F \longrightarrow E$ linear, instead of a direct minimisation of the functional $j$ in the space $E$ of the control variables, the gradient algorithm is applied for the minimisation of $j \circ P$ in $F$. Furthermore, the resulting algorithm remains a descent method in the space $E$. In the context of a discrete gradient computation in aerodynamic shape design, a practical example can be to consider as control variables the ordinates of the $m$ shape grid-points, i.e. take $E=\mathbb{R}^{m}$.

Different spaces $F$ are then considered, each of them corresponding to a subset of points extracted from the complete parametrisation. For each level $l$, a linear application $P^{(l)}: F=\mathbb{R}^{n_{l}} \longrightarrow E=\mathbb{R}^{m}$ is defined allowing to prolonged the $n_{l}$ parameters on the finest level. Then, at iteration $r$ and level $l$, the control variables are updated as follows:

$$
\gamma_{r+1}=\gamma_{r}-\omega_{r} P^{(l)}\left(P^{(l)}\right)^{*} g_{r} \quad \text { with } \omega_{r} \in \mathbb{R} \text { and } g_{r} \in \mathbb{R}^{m},
$$

$g_{r}$ being the gradient of the cost functional at iteration $r$, and, $\left(P^{(l)}\right)^{*}$ the adjoint $P^{(l)}$ adjoint, which is associated to the transpose of the matrix $M^{(l)} \in \mathbb{R}^{m \times n_{l}}$ relative to $P^{(l)}$. Note that, only the differentiation of the cost functional with respect to the complete parametrisation is needed since the minimisation on the coarser levels appears uniquely through the matrix $M^{(l)}\left(M^{(l)}\right)^{T} \in \mathbb{R}^{m \times m}$. Thus, this algorithm can be also interpreted as a preconditioned gradient method. On another hand, the level choice at each optimisation iteration is determinate by a strategy of level changes similar to multilevel/multigrid strategies used for the resolution of partial differential equations (as, for instance, V-cycles). The particular algorithm is then totally definite by the choice of the operator $P^{(l)}$. For shape-grid points as control parameters, a natural way to define the prolongation operator is to use interpolations. Different interpolations have been considered in [2] giving convergence curves sensibly different. Finally, a Hermitian cubic interpolation coupled with a set of embedded subparametrisations (for each increase of level, the number of points is doubled), has been chosen in [2], and, also used in successive works (see, e.g. [5]).

Concerning the discrete gradient computation, both adjoint-based and divided-differences sensitivities can be used. Adjoint approaches allow to compute an exact gradient and to highly reduce the number of flow evaluations requisite by the divided differences. Nevertheless, the classical approximate gradient based on divided differences remains of interest as far as the exact differentiation is a too complex task. Note that the computational cost can be noticeably reduced by coupling with a multilevel approach in which the divided differences are applied on the coarser levels (see, e.g. [2]). In this case, the control variables are updated as 
follows:

$$
\text { for } i=1, \cdots, m \quad\left(\gamma_{r+1}\right)_{i}=\left(\gamma_{r}\right)_{i}-\omega_{r} \sum_{k=1}^{n_{l}}\left(M^{(l)}\right)_{i k} \frac{1}{\epsilon}\left[j\left(\gamma_{r}+\epsilon P^{(l)}\left(e_{k}\right)\right)-j\left(\gamma_{r}\right)\right]
$$

in which $e_{k}$ is the $k$-th element of the canonical basis of $\mathbb{R}^{n_{l}}, \epsilon$ an adequate small given parameter.

Furthermore, in [5], this multilevel/divided-differences formulation has been also used in a one-shot approach [9] in which the flow equations are progressively solved. In practical, at each optimisation iteration, the flow evaluations are done considering only a very partial resolution of the steady governing equations (only few pseudo-time steps using in the pseudo-unsteady approach and few iterations of the iterative method for solving the linear system involved in the implicit linearised scheme).

\section{Multilevel method associated to BÉzier parametrisation}

A Bézier curve of degree $n$ can be defined according to its parametrisation, i.e.:

$$
S(t)=(x(t), y(t))=\sum_{q=0}^{n} B_{n}^{q}(t) S_{q} \quad \text { with } t \in[0,1]
$$

where $S_{q}=\left(x_{q}, y_{q}\right)$ is the $q$-th Bézier control point while $B_{n}^{q}(t)$ corresponds to the $q$-th Bernstein polynomial. The ordinates of the shape grid-points, i.e. $\gamma=\left(y_{0}^{\Gamma}, \cdots, y_{m}^{\Gamma}\right)^{T}$, are here considered as design variables. Furthermore, the parameters $\left(t_{k}\right)_{k=0, m}$ being given $\left(t_{0}=0<t_{1}<\cdots<t_{m}=1\right)$ and using relation $(3)$ with $y\left(t_{k}\right)=y_{k}^{\Gamma}$ for $k=0, \cdots, m$, it clearly appears that each control variable can be expressed as a linear combination of the ordinates of the Bézier control points $\alpha=\left(y_{0}, \cdots, y_{n}\right)^{T}$. Thus, the following linear operator from $\alpha$ to $\gamma$ can be considered:

$$
\begin{aligned}
& P: \mathbb{R}^{n+1} \longrightarrow \mathbb{R}^{m+1} \\
& \alpha \quad \longmapsto \gamma=P(\alpha)=M \alpha \quad \text { where } M_{i j}=B_{n}^{j}\left(t_{i}\right) .
\end{aligned}
$$

Then, thanks to the linearity of $P$, it is possible to define a strategy similar to the original multilevel approach [2], where instead of a subset of boundary grid-points, each subparametrisation is a set of Bézier control points. In this case, the following algorithm can be defined:

$$
\gamma_{r+1}=\gamma_{r}-\omega_{r} d_{r}^{(l)} \text { with } \omega_{r} \in \mathbb{R} \text { and } \gamma_{r}, \gamma_{r+1} \text { and } d_{r}^{(l)} \in \mathbb{R}^{m+1}
$$

in which the descent direction at iteration $r$ and level $l$ is defined by:

$$
k=0, \cdots, m \quad\left(d_{r}^{(l)}\right)_{k}=\sum_{j=0}^{m}[\underbrace{\sum_{q=0}^{n_{l}} B_{n_{l}}^{q}\left(t_{k}^{(l)}\right) B_{n_{l}}^{q}\left(t_{j}^{(l)}\right)}_{\left(M^{(l)}\left(M^{(l)}\right)^{T}\right)_{k j}}]\left(g_{r}\right)_{j}
$$

Nevertheless, the definition of the subparametrisations is, here, not so obvious. Indeed, the abscissae of shape grid-points $\left(\left\{x_{k}^{\Gamma}\right\}_{k=0, m}\right)$ being given, at each level, $X^{(l)}=\left(x_{0}^{(l)}, \cdots, x_{n_{l}}^{(l)}\right)^{T}$ with $n_{l}>n_{l-1}$ and $T^{(l)}=$ $\left(t_{0}^{(l)}, \cdots, t_{m}^{(l)}\right)^{T}$ should be defined such that $(3)$ be always verified, i.e.:

$$
x_{k}^{\Gamma}=x\left(t_{k}^{(l)}\right)=\sum_{q=0}^{n_{l}} B_{n_{l}}^{q}\left(t_{k}^{(l)}\right) x_{q}^{(l)} \text { for } k=0, \cdots, m
$$

Let us now suppose that the parametrisation on the coarsest level has been yet defined with $X^{(0)}$ and $T^{(0)}$ consistent, i.e. with (5) verified for $k=0$. For instance, if, as for the case of the nozzle inverse problem presented in Sec. 3, the $\left(x_{k}^{\Gamma}\right)_{k=0, m}$ are uniformly distributed, then a consistent coarsest level can be easily obtained by 

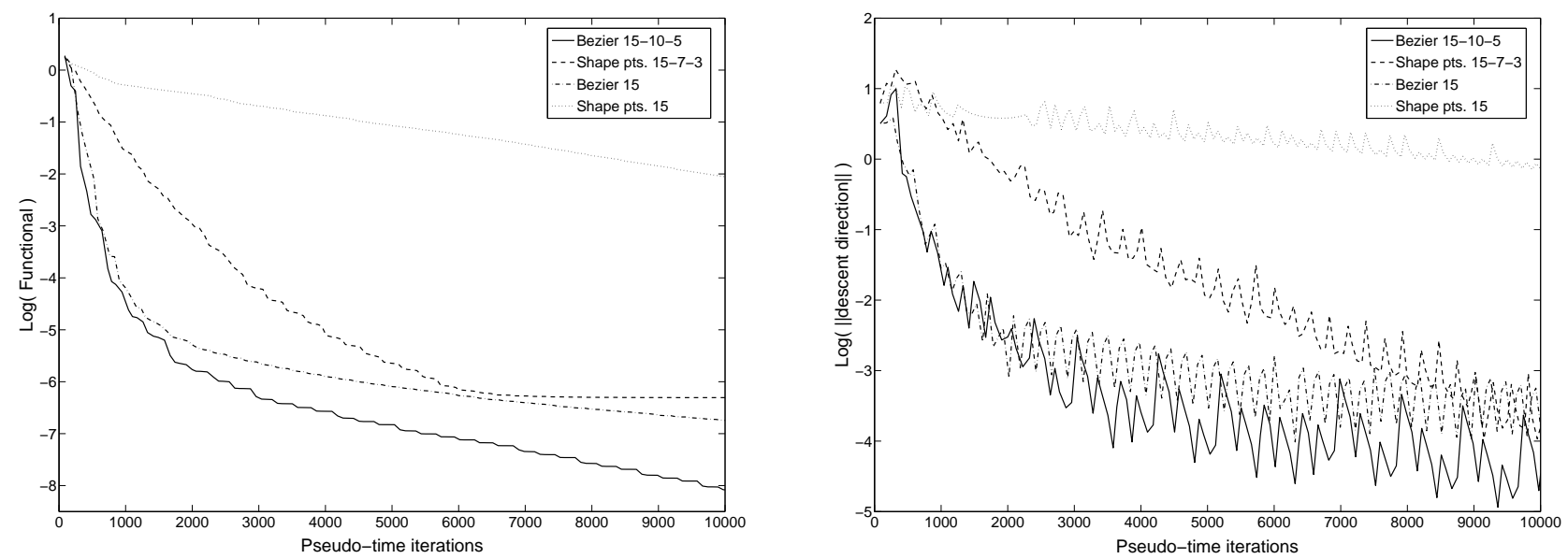

Figure 1. Convergence histories for $j\left(\gamma_{r}\right)$ (left) and $d_{r}^{(l)}$ (right) in the case of an adjoint gradient computation: comparison between shape grid-points and Bézier parametrisations for both on one level (15 parameters) and a V-cycle multilevel approach on three levels.

simply choosing an uniform distribution for both $X^{(0)}$ and $T^{(0)}$. In order to define a parametrisation on the other levels, the property of degree elevation of the Bézier curves is used here. Indeed, the degree-elevation property allows to increase the degree and the number of control points of a Bézier curve without any change on the distribution of the parameters $t$ (see, e.g. [4]). Then, the parameters $\left\{t_{k}\right\}_{k=0, m}$ keep unchanged on all the levels, i.e. $t_{k}^{(l)}=t_{k}^{(0)} \equiv t_{k}$ for $k=0, \cdots, m$ et $l>0$, while $X^{(l)}$ for $l>0$ is obtained applying successively the degree-elevation algorithm until obtaining $n_{l}+1$ abscissae. More precisely, the following algorithm is considered to obtain $X^{(l)}$ from $X^{(0)}$ (with the convention $x_{-1}=0$ ):

$s \longleftarrow 1$

For $q=0, \cdots, n_{0} \quad x_{q} \longleftarrow x_{q}^{(0)}$

Repeat until $s=l$

Obtain $X^{(s)}$ from $X^{(s-1)}$ as follows:

$n \longleftarrow n_{s-1}$

Repeat until $n=n_{s}$

$x_{q} \longleftarrow \frac{q}{n+1} x_{q-1}+\left(1-\frac{q}{n+1}\right) x_{q} \quad$ for $q=0, \cdots, n+1$

$n \longleftarrow n+1$

$s \longleftarrow s+1$

$X^{(l)} \longleftarrow\left(x_{0}, \cdots, x_{n}\right)^{T}$

\section{A NUMERICAL EXAMPLE: A 2D NOZZLE INVERSE PROBLEM}

Let us consider a classical test-case already used for the multilevel approach associated to shape grid-point coordinates parametrisation $[2,5]$. It is a $2 \mathrm{D}$ convergent-divergent nozzle inverse problem for inviscid subsonic flows (the flow is modelled, here, by the Euler equations). The corresponding cost functional is expressed as a boundary integral which involves the flow variables through the pressure field. An initial constant-section nozzle and a target sine shape are considered while the computational mesh is composed, here, of 423 nodes with 31 points on the upper boundary of the throat nozzle, i.e. where the shape should be optimised.

Fig. 1 shows the convergence behaviour obtained with different strategies when the gradient is computed by an adjoint approach. The convergence is expressed with respect to the number of pseudo-time iterations. 

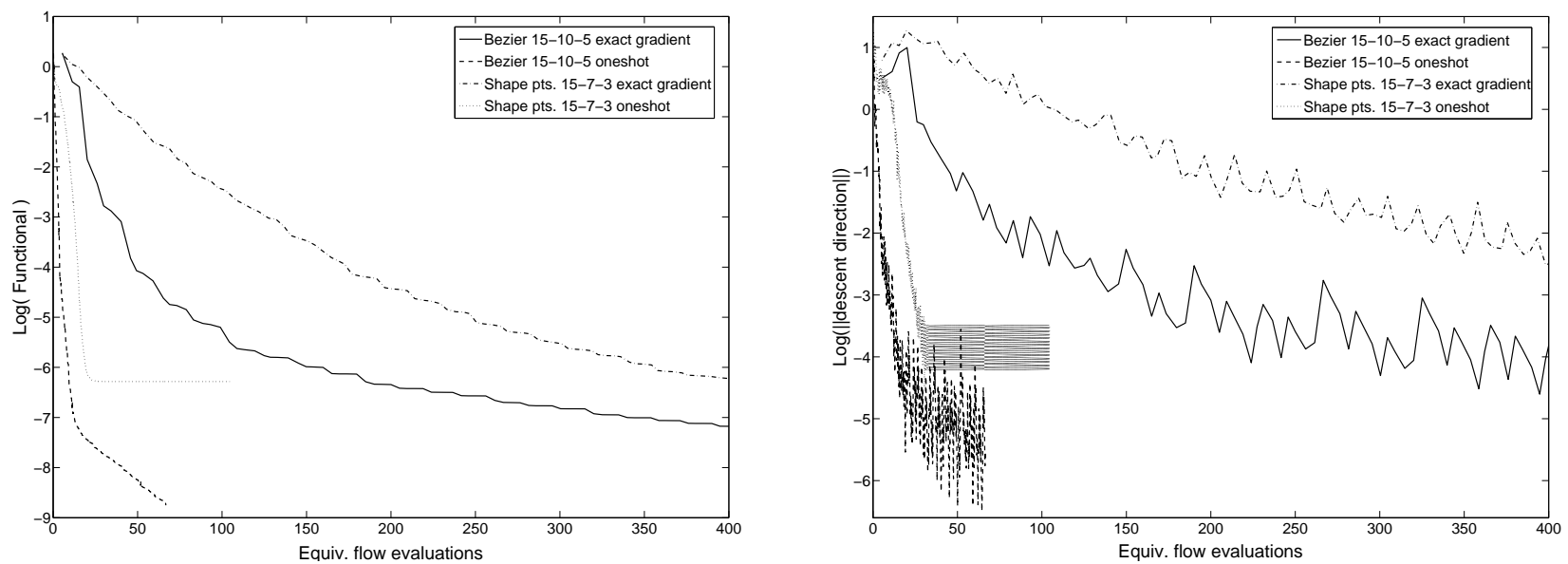

Figure 2. Convergence histories for $j\left(\gamma_{r}\right)$ (left) and $d_{r}^{(l)}$ (right): comparison between exact adjoint gradient computation and gradient approximate by divided differences associated with a one-shot approach.
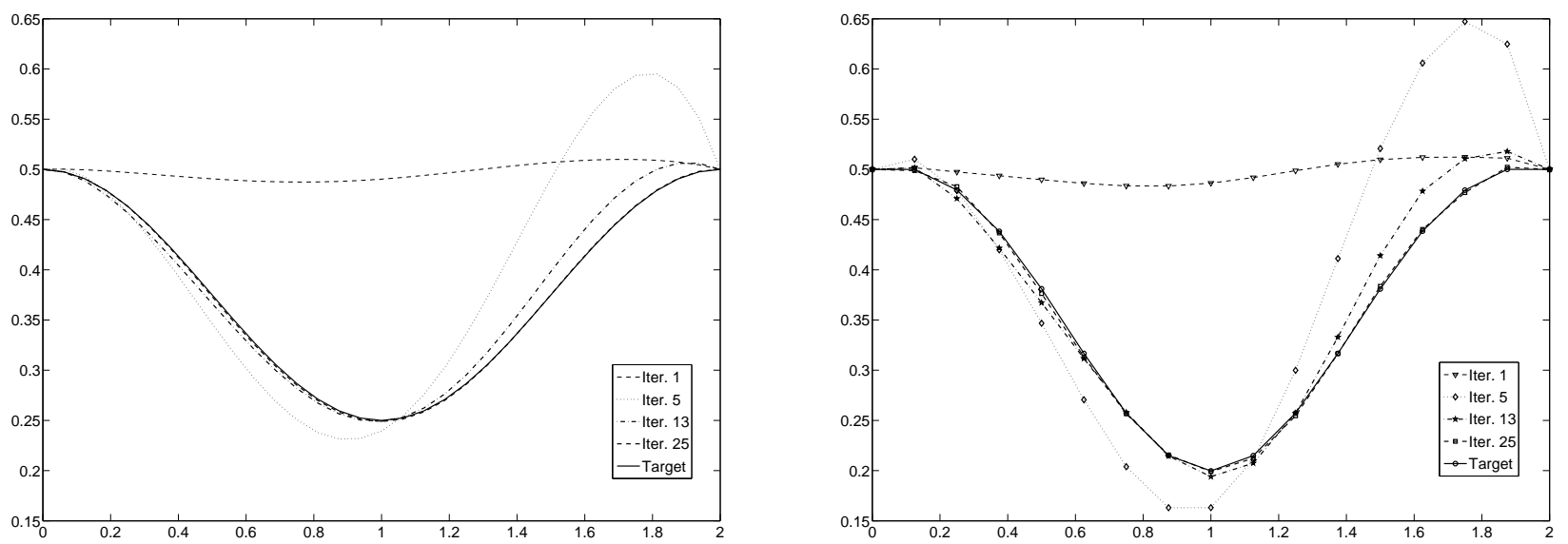

FiguRE 3. One-shot/multilevel approach using Bézier parametrisation: successive shapes (left) and Bézier control points (right). Comparison between the target solution and solutions after 1, 2, 4 and $7 \mathrm{~V}$-cycles (i.e., after 1, 5, 13 and 25 optimisation iterations).

Indeed, at each optimisation iteration, different flow evaluations are needed (one for the adjoint gradient and few others for the 1D search of an optimal $\omega_{r}$ ), each of them requiring a loop in time since the steady governing equations are solved, here, by using a pseudo-unsteady approach. For the original parametrisation based on shape grid-points, a large convergence improvement is obtained considering a V-cycle multilevel approach with three levels (3, 7 and 15 design variables) instead of a one-level approach using 15 control parameters, i.e. already with a smoothing since 31 variables are available. Concerning the new set of subparametrisation based on Bézier control points as introduced in Sec. 2, even the one-level approach (15 control parameters) shows better results than the previous multi-level approach. The corresponding multilevel strategy with three levels (5, 10 and 15 design variables) yields ulterior improvements, even if the multilevelling appears less impressive. Note that the same number of parameters on the finest sublevel has been taken for the two kinds of subparametrisation. 
Nevertheless, it does not correspond to the same level of shape description since the Bézier control points allow a more compact representation than the shape grid-points.

Fig. 2 reports the same V-cycle three-levels approaches applied both for the adjoint method and the divideddifferences one associated with a one-shot approach. The good behaviour of the one-shot approach without adjoint, already obtained in [5], is also confirmed for the subparametrisation based on Bézier curves. Moreover, the same improvement obtained by using the Bézier parametrisation instead of the shape grid-points is observed with the multilevel/divided-differences one-shot approach. Fig. 3 shows the shape evolution during the optimisation process for the one-shot method with the Bézier parametrisation and the multilevel/divided-differences formulation. Note that, the cost for obtaining a converged flow solution corresponds approximatively to two V-cycles on three levels (5, 10 and 15 parameters) with the one-shot approach (i.e., here only one pseudo-time iteration and three iterations of the iterative method for solving the linear system). Thus, with a computational cost equivalent to four flow evaluations, we already obtain a shape very close to the target one. Fig. 3 also shows on the right frame, the evolution of the Bézier control points. In this simple case, even if we consider an uniform distribution of the abscissae, the resulting distribution of the ordinates of the Bézier control points is very regular without any oscillation. Thus, an adaptation of the parametrisation as proposed in [1] is not needful, here, at least in this particular case.

\section{Conclusion}

In the present study, a multilevel gradient-based method for optimum shape design in aerodynamics is described. This approach starts from an existing formulation [2] based on an embedded parametrisation of shape grid-points and on interpolation operators. A new set of subparametrisations is then proposed, in which a coarse level is described by using Bézier control points while the prolongation operator is obtained through the application of the degree elevation formula. Thus, this approach seems not so far from the one proposed in [4]. Nevertheless, contrary to [4], a descent direction method is always considered, and moreover, the control variables on the finest level are still the ordinates of the shape grid-points. Some numerical experiments have been done on a simple $2 \mathrm{D}$ nozzle inverse problem showing that the introduction of this new family of subparametrisations has suitable effects on the convergence behaviour. This is true considering an adjoint formulation as well as an approximate gradient associated to a one-shot method. Note that, since the Bézier curves act as a basic tool for polynomial shape representation, one can also envisage to extend the formulation to more complex curves as B-splines, and also, to 3D case through, for instance, tensorial Bézier parametrisation.

\section{REFERENCES}

[1] B. Abou El Majd, J.-A. Désidéri and A. Janka, Nested and self-adaptive Bézier parameterization for shape optimization, International Conference on Control, Partial Differential Equations and Scientific Computing, Beijing, China, 13-16 Sept, 2004 .

[2] F. Beux and A. Dervieux, A hierarchical approach for shape optimization, Engineering Computations, 11/1, 25-48, 1994.

[3] F. Courty and A. Dervieux, Multilevel functional Preconditioning for shape optimisation, International Journal of Computational Fluid Dynamics, 20/7, 481-490, 2006.

[4] J.-A. Désidéri, Hierarchical optimum-shape algorithms using embedded Bézier parameterizations, Numerical Methods for Scientific Computing, Variational Problems and Applications, E. Heikkola, Y. Kuznetsov, P. Neittaanmäki and O. Pironneau et al eds., CIMNE, chapter Hierarchical Optimum-Shape Algorithms Using Embedded Bézier Parameterizations, 2003.

[5] C. Held and A. Dervieux, One-shot airfoil optimisation without adjoint, Computers and Fluids, 31/8, 1015-1049, 2002.

[6] B. Mohammadi and O. Pironneau, Applied Shape Optimization for Fluids, Numerical Mathematics and Scientific Computation, Oxford University press, 2001.

[7] E. Polak, Optimization: algorithms and consistent approximations, Applied Mathematical Sciences, 124, Springer-Verlag, New York, 1997.

[8] J. Reuther and A. Jameson, Aerodynamic shape optimization of wing and wing-body configurations using control theory, AIAA Paper, 95-0123, 1995.

[9] G. Kuruvila, S. Ta'asan and M.D. Salas, Airfoil optimization by the one-shot method, Von Karman Lecture Series, 1994. 